\title{
LES SENSILLES DE LA CERCAIRE D'HAPLORCHIS TAICHUI (Digene, Heterophyidae).
}

\section{Discussion sur la systématique des Opisthorchioidea}

\author{
Ch. BAYSSADE-DUFOUR*, J.-L. ALBARET* et C. K. OW-YANG**
}

\begin{abstract}
RÉSUMÉ. La chétotaxie cercarienne d'Haplorchis taichui (Haplorchiinae, Heterophyidae) est décrite et comparée à celle de dix autres cercaires d'Opisthorchioidea dont trois appartiennent aux Acanthostomidae (Acanthostominae), une aux Opisthorchiidae (Opisthorchiinae) et six aux Heterophyidae, parmi lesquels on compte deux Centrocestinae, deux Apophallinae, un Euryhelminthinae et un Haplorchïnae.

La chétotaxie de la cercaire permet de différencier les espèces et les genres considérés; elle ne reflète pas la division en familles basée sur la morphologie des adultes et elle plaide en faveur de l'hétérogénéité des Heterophyidae; elle permet de caractériser la superfamille des Opisthorchioidea par l'absence de symétrie hexaradiée au niveau du premier cycle de papilles céphaliques et par la présence de deux à cinq paires de papilles U sur une queue pourvue de pores excréteurs proximaux.
\end{abstract}

\section{Sensillae of cercariae of Haplorchis taichui (Digenea, Heterophyidae). Discussion of the systematics of the Opisthorchioidea.}

SUMMARY. Cercarial chaetotaxy of Haplorchis taichui (Haplorchiinae, Heterophyidae) is described and compared to that of ten other Opisthorchioidea : three belonging to the Acanthostomidae (Acanthostominae), one to the Opisthorchiidae (Opisthorchiinae) and six to the Heterophyidae (among these, two Centrocestinae, two Apophallinae, one Euryhelminthinae and one Haplorchiinae).

Cercarial chaetotaxy allow us to distinguish the considered species and genera, but does not support division of the Opisthorchioidea into families as these have been defined based on morphology of adults; it suggests that the Heterophyidae is heterogenous; it allows us to recognize the Opisthorchioidea characterized by a loss of hexaradial symmetry on the first cephalic cycle of sensillae and by the presence of two to five pairs of sensillae on a tail with proximal excretory apertures.

\footnotetext{
* Laboratoire des Vers, Muséum National d'Histoire Naturelle, 61 rue Buffon, F 75231 Paris Cedex 05.

** Institut Penyelidikan Perubatan (Institute for Medical Research) Jalan Pahang, Kuala Lumpur 02-14 Malaysia.
}

Accepté le 3 novembre r982. 
La division des Opisthorchioidea en familles repose sur des caractères morphologiques de Vers adultes mais les cercaires correspondantes sont difficiles à différencier d'une famille à l'autre et, selon La Rue (1957), leurs seules caractéristiques sont celles du sous-ordre des Opisthorchiata : " pores excréteurs sur les bords latéraux de la queue, près de la jonction de celle-ci avec le corps ». La chétotaxie permet, d'ordinaire, la détermination d'un Trématode au stade cercarien à tous les niveaux de la systématique, de la variation infraspécifique à la division en super-ordres.

Il est donc particulièrement intéressant de tester la valeur de ce caractère aux différents niveaux taxonomiques, dans le cas des Opisthorchioidea.

Nous décrivons la chétotaxie de la cercaire d'Haplorchis taichui (Nishigori, 1924), la comparons à celle d'Haplorchis pumilio et à celles de neuf autres cercaires d'Opisthorchioidea.

Le lot de cercaires d'Haplorchis taichui provient d'un Mollusque Prosobranche Melaniidae: Melanoides tuberculata naturellement infesté de Segambut dans la région de Kuala Lumpur en Malaisie ; les métacercaires et les adultes correspondants ont été obtenus expérimentalement chez un Poisson et un chat; la morphologie des stades successifs est identique à celle décrite par Nishigori (1924) et Faust et Nishigori (1926) à Formose (Chine) chez Haplorchis taichui dont le cycle admet pour hôtes sucessifs : Melania obliquegranosa, plusieurs Poissons et plusieurs carnivores dont l'Homme ; il s'agit donc, soit de la même espèce, soit d'une espèce indifférenciable par les critères habituels.

\section{I - Description des sensilles d'Haplorchis taichui}

Les sensilles ont été mises en évidence par imprégnation au nitrate d'argent selon la méthode de Combes et coll. (1976) ; elles se répartissent sur la tête, le corps et la queue.

Chétotaxie céphalique (fig. 1, D. E)

La tête porte 76 à 78 sensilles soit de part et d'autre du plan médio-sagittal :

a) sensilles buccales

$$
\begin{aligned}
& \mathrm{C}_{\mathrm{I}}=6 \text { sensilles } \\
& \mathrm{C}_{\mathrm{II}}=3 \mathrm{C}_{\mathrm{II}} 1,1 \mathrm{C}_{\mathrm{II}} 2,3 \mathrm{C}_{\mathrm{II}} 3,3 \mathrm{C}_{\mathrm{II}} 4 \\
& \mathrm{C}_{\mathrm{III}}=2 \mathrm{C}_{\mathrm{III}} 1,2 \mathrm{C}_{I I I} 2,2 \mathrm{C}_{I I I} 3,1 \mathrm{C}_{\mathrm{III}} 4
\end{aligned}
$$

b) sensilles dorso-céphaliques

$$
\mathrm{H}=10 \text { à } 11 \mathrm{H}_{1}, 1 \mathrm{H}_{2}, 2 \mathrm{H}_{3}, 2 \mathrm{HL}_{3}
$$

Chétotaxie corporelle (fig. 1, A-C, fig. 2, A-D)

Elle compte 64 à 76 sensilles, soit par hémicorps

$$
\begin{aligned}
& \mathrm{A}_{\mathrm{I}}=1 \mathrm{~A}_{\mathrm{I}} \mathrm{V}, 3 \text { ou } 4 \mathrm{~A}_{\mathrm{I}} \mathrm{L} \\
& \mathrm{A}_{\mathrm{II}}=1 \mathrm{~A}_{\mathrm{IIV}} \mathrm{V}, 3 \text { ou } 4 \mathrm{~A}_{I I} \mathrm{~L}, 2 \mathrm{~A}_{I I} \mathrm{D} \\
& \mathrm{A}_{I I I}=4 \mathrm{~A}_{I I I} \mathrm{~V}, 1 \mathrm{~A}_{I I I} \mathrm{~L}
\end{aligned}
$$




$$
\begin{aligned}
& \mathrm{M}=2 \mathrm{MV}, 2 \mathrm{ML}, 1 \mathrm{MD} \\
& \mathrm{P}_{\mathrm{I}}=2 \mathrm{P}_{\mathrm{I}} \mathrm{V}, 3 \text { ou } 4 \mathrm{P}_{\mathrm{I}} \mathrm{L} \\
& \mathrm{P}_{\mathrm{II}}=3 \text { à } 5 \mathrm{P}_{\mathrm{II}} \mathrm{L} \\
& \mathrm{P}_{I I I}=1 \mathrm{P}_{\text {III }} \mathrm{L}, 1 \mathrm{P}_{I I I} \mathrm{D} \\
& \mathrm{P}_{I V}=1 \mathrm{P}_{I V} \mathrm{~V}, 0 \text { ou } 1 \mathrm{P}_{I V} \mathrm{~L}, 1 \mathrm{P}_{I V} \mathrm{D}
\end{aligned}
$$
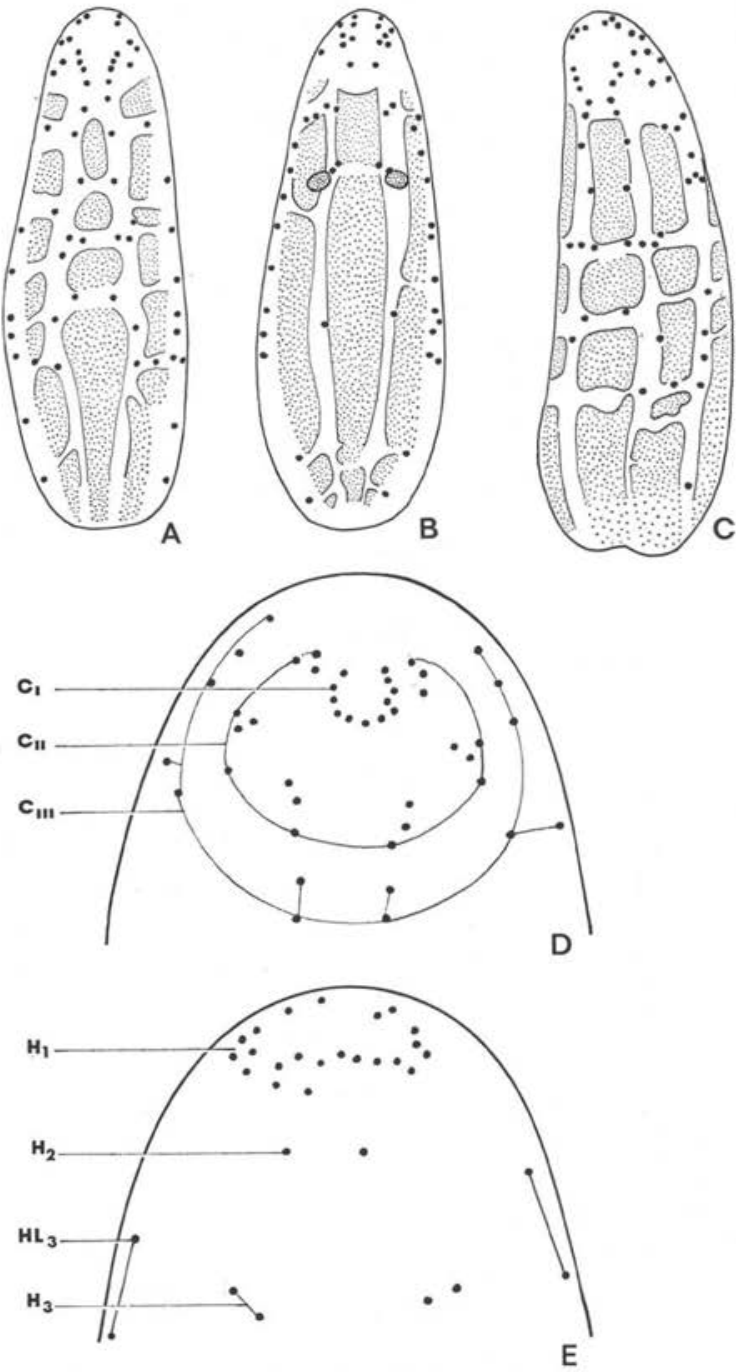

FIG. I, A, B, C. - Distribution des sensilles sur les commissures et les axes nerveux de la cercaire d'Haplorchis taichui; à l'imprégnation argentique, les commissures et axes nerveux se détachent en clair sur le fond pigmenté du corps.

$A$ : vue ventrale ; $B$ : vue dorsale ; $C$ : vue latérale; $D, E$ : chétotaxie céphalique de la cercaire d'Haplorchis taichui; D : vue ventrale ; E : vue dorsale. 

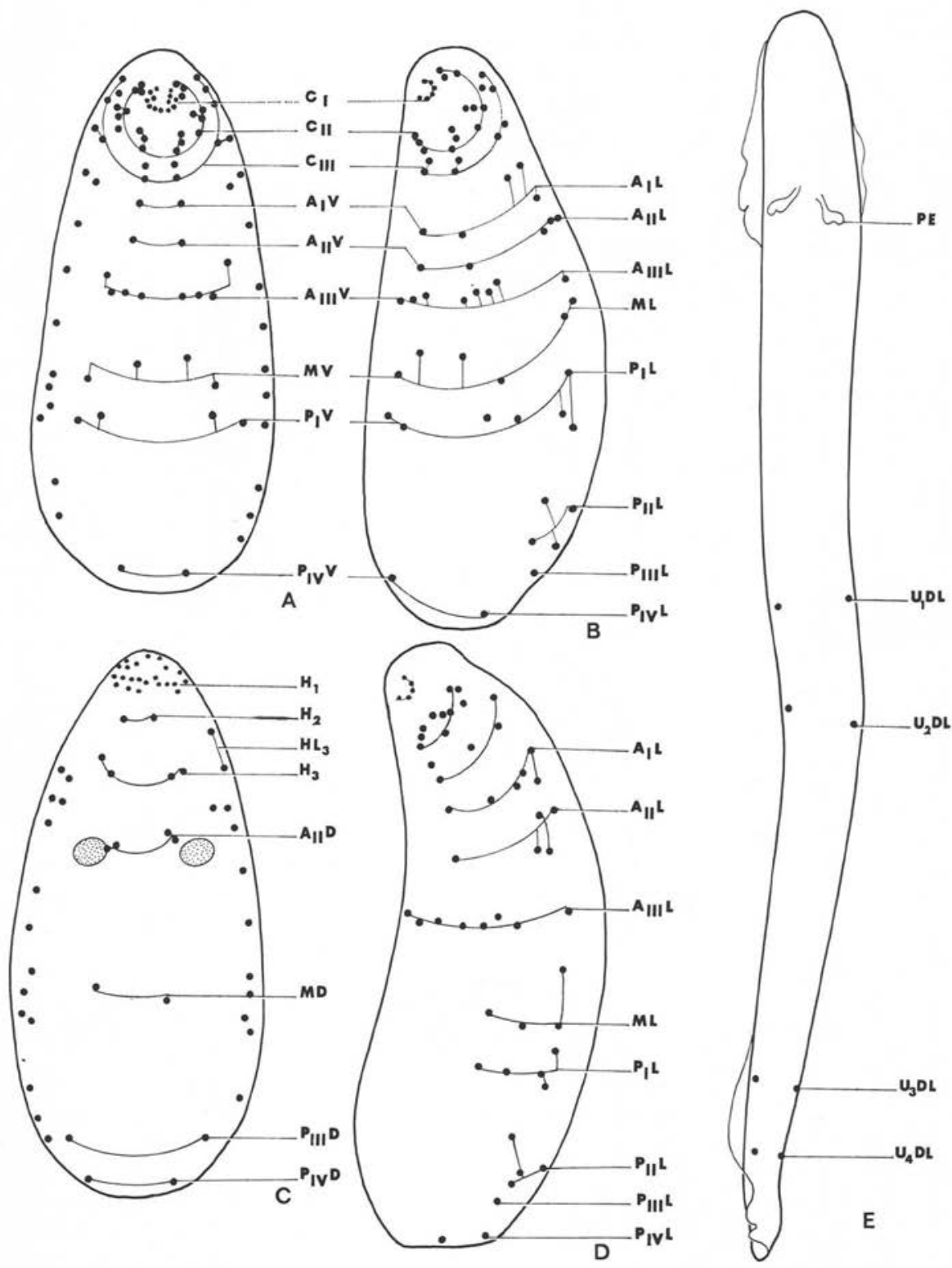

Fig. 2. - Chétotaxie céphalique,

corporelle et caudale de la cercaire d'Haplorchis taichui.

A, B, D : Sensilles céphaliques et corporelles ventrales et latérales ; C : Sensilles céphaliques et corporelles dorsales et latérales; $\mathrm{E}$ : Sensilles caudales (U) et pores excréteurs (P. E). 
Chétotaxie caudale (fig. 2 E) ; nombre total de sensilles

$\mathrm{U}=4$ paires de $\mathrm{UDL}$ soit $2 \mathrm{U}_{1} \mathrm{DL}, 2 \mathrm{U}_{2} \mathrm{DL}, 2 \mathrm{U}_{3} \mathrm{DL}, 2 \mathrm{U}_{4} \mathrm{DL}$

\section{II - Comparaison avec Haplorchis pumilio (fig. $3 \mathrm{~A}, \mathrm{~B}$ )}

La chétotaxie d'Haplorchis pumilio a été décrite par Bayssade-Dufour et OwYang (1975). Haplorchis taichui et $H$. pumilio montrent :

1 - plusieurs niveaux semblables: $\mathrm{C}_{I I} 2, \mathrm{C}_{I I I} 3, \mathrm{~A}_{\mathrm{I}} \mathrm{V}, \mathrm{A}_{\mathrm{II}} \mathrm{V}, \mathrm{MV}, \mathrm{P}_{\mathrm{I}} \mathrm{V}, \mathrm{P}_{\mathrm{III}} \mathrm{D}, \mathrm{P}_{\mathrm{IV}} \mathrm{D}$, $\mathrm{U}_{1} \mathrm{DL}, \mathrm{U}_{2} \mathrm{DL}, \mathrm{U}_{3} \mathrm{DL}$.
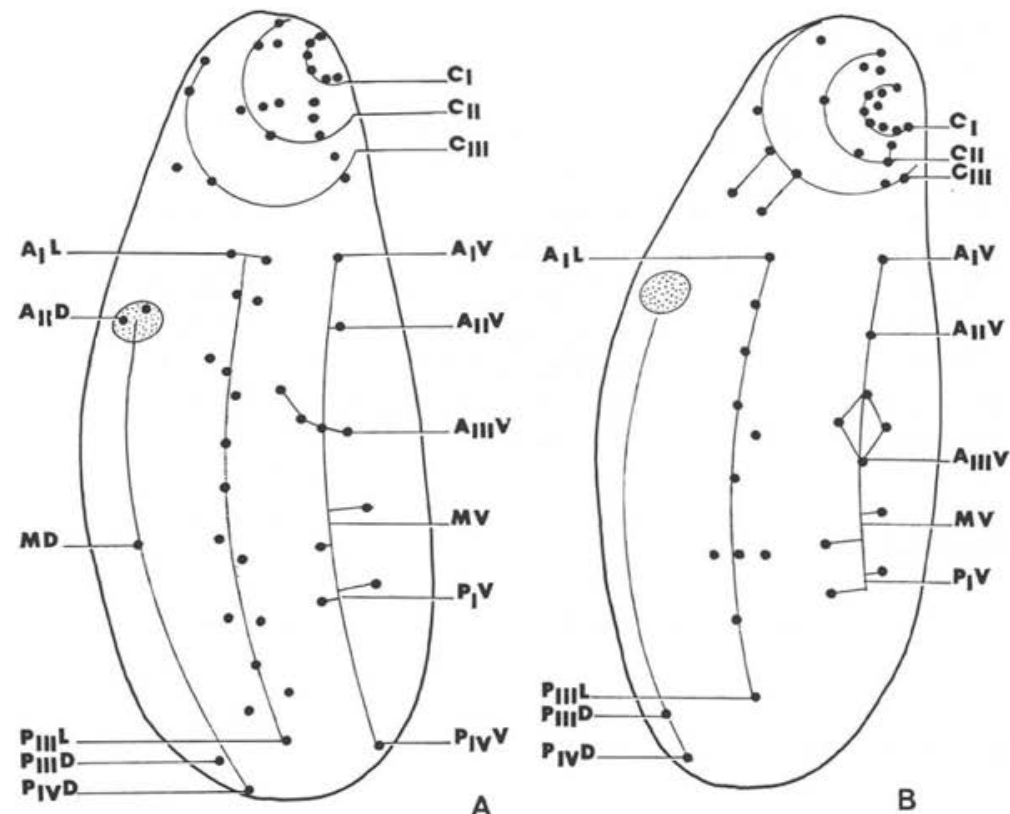

FIG. 3, A, B. - Chétotaxies comparées d'Haplorchis taichui (A) et Haplorchis pumilio (B).

2 - plusieurs niveaux peu différents avec des nombres de papilles identiques mais une autre disposition : $\mathrm{C}_{\mathrm{III}} 1$ alignées verticalement chez $H$. taichui, horizontalement chez $H$. pumilio, $\mathrm{C}_{\mathrm{III}} 3$ et 4 groupées chez $H$. taichui, séparées chez $H$. pumilio, $\mathrm{A}_{\mathrm{III}} \mathrm{V}$ disposées selon une ligne brisée ouverte chez $H$. taichui, fermée chez $H$. pumilio, $\mathrm{U}_{4}$ dorso-latérale chez $H$. taichui, dorsale chez $H$. pumilio.

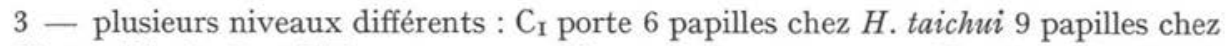
$H$. pumilio, $\mathrm{A}_{\mathrm{II}} \mathrm{D}$ et $\mathrm{MD}$ portent respectivement deux et une papilles chez $H$. taichui, 
n'en portent pas chez $H$. pumilio, les niveaux AL-PL totalisent 16 à 22 papilles chez H. taichui, 9 à 15 chez H. pumilio.

Les niveaux chétotaxiques énumérés en 1) et 2) sont communs aux deux espèces du genre Haplorchis ; les niveaux énumérés en 3) permettent de séparer les espèces taichui et pumilio.

\section{III - Comparaison avec les autres chétotaxies cercariennes d'Opisthorchioidea}

\section{A - Documents utilisés}

Outre Haplorchis pumilio et Haplorchis taichui (Haplorchiinae) nous connaissons les chétotaxies cercariennes de :

- cinq autres Heterophyidae : Apophallus muehlingi et A. donicus (Apophallinae) Euryhelmis squamula (Euryhelminthinae) Centrocestus formosanus et Centrocestus sp. (Centrocestinae) respectivement décrits par Odening (1973), Combes, Jourdane et Richard (1974), Bayssade-Dufour, Albaret et Ow-Yang (1982),

- trois Acanthostomidae : Timoniella (Acanthostomum) imbutiforme, Timoniella praeterita et Aphalloides coelomicola (Acanthostominae) respectivement décrits par Maillard (1973), Bayssade-Dufour (1979), Bayssade-Dufour et Maillard (1982),

- un Opisthorchiidae : Clonorchis sinensis (Opisthorchiinae) décrit par BayssadeDufour, Albaret et Dong Cham Kim (1982).

\section{B - Résultats}

1 - La chétotaxie permet la détermination spécifique dès le stade cercarien.

Chacune des cercaires nommées ci-dessus a une chétotaxie bien particulière qui permet de l'identifier dès ce stade et de la reconnaître d'une espèce même proche alors que sa seule anatomie ne le permet pas (cf. auteurs cités plus haut).

2 - La chétotaxie permet la détermination générique.

Les genres Apophallus, Centrocestus, Haplorchis et Timoniella sont représentés chacun par deux espèces; nous constatons des différences très sensibles entre les quatre genres et, au contraire, une grande similitude chétotaxique à l'intérieur de chacune des paires d'espèces (cf. auteurs cités plus haut).

3 - La chétotaxie ne permet pas la détermination des trois familles considérées : Acanthostomidae, Heterophyidae et Opistorchiidae.

Jusqu'à maintenant, dès qu'un nombre suffisant de cercaires a été étudié, il a toujours été possible de trouver des éléments caractéristiques de chaque famille en utilisant essentiellement les niveaux $C_{I}$ et $U$. 

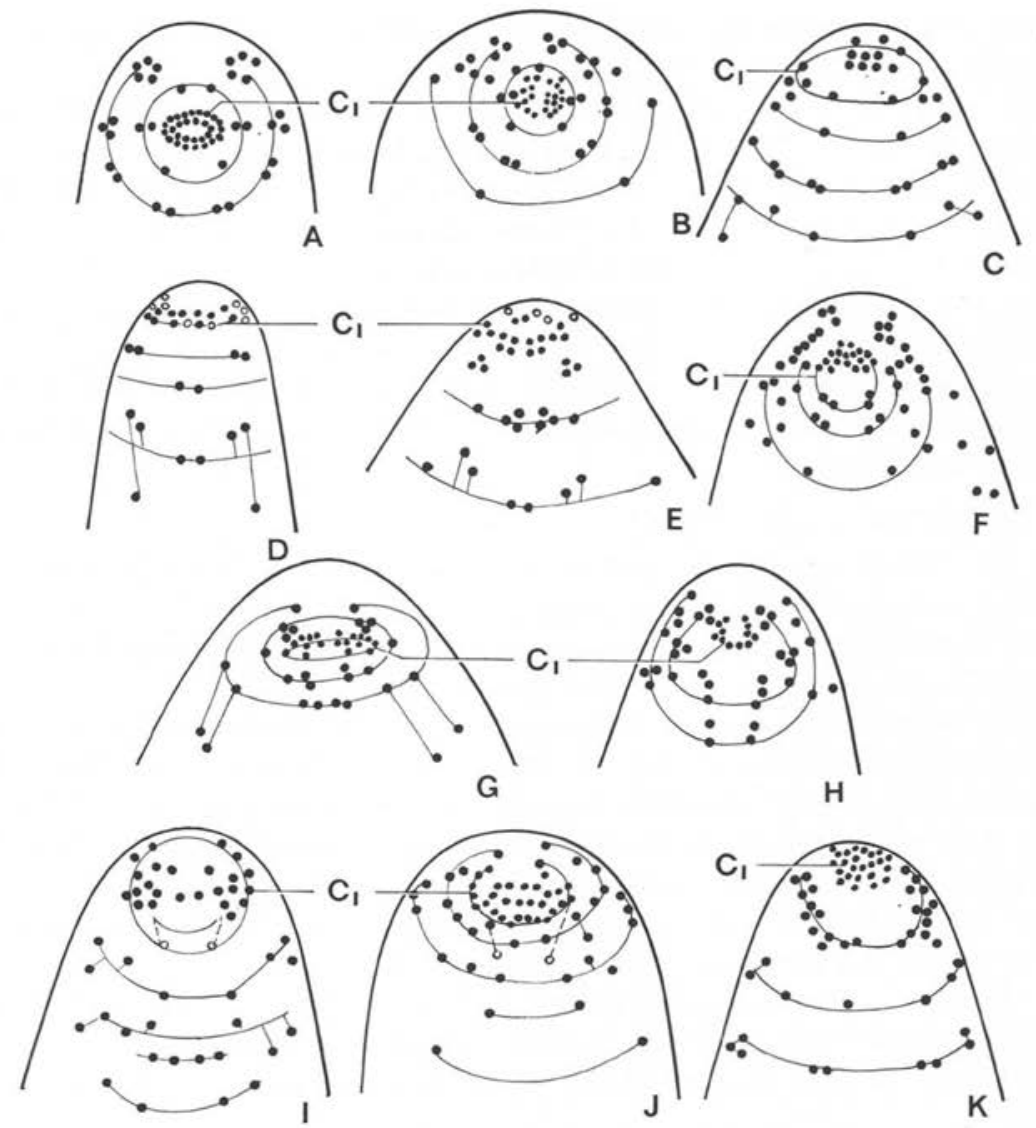

$\mathrm{H}$

Fig. 4. - Chétotaxies céphaliques de cercaires d'Opisthorchioidea.

A : Timoniella imbutiforme d'après Maillard (1973) ; B : Timoniella praeterita ; C : Aphalloides coelomicola d'après Maillard (1976) ; D et E : A pophallus muehlingi et Apophallus donicus d'après Odening (I973); F : Euryhelmis squamula d'après Combes, Jourdane et Richard (1974); G : Haplorchis pumilio d'après Bayssade-Dufour et Ow-Yang (1975); H : Haplorchis taichui; I et $\mathrm{J}$ : Centrocestus formosanus et Centrocestus sp. d'après Bayssade-Dufour, Albaret et Ow-Yang (1982) ; K : Clonorchis sinensis d'après Bayssade-Dufour, Albaret et Dong Cham Kim (1982) : en $\mathrm{D}$ et $\mathrm{E}$, les papilles représentées en clair sont dorsales.

Nous avons donc considéré ces niveaux chez les onze cercaires d'Opisthorchioidea dont trois sont attribuées aux Acanthostomidae, sept aux Heterophyidae et une aux Opisthorchiidae.

\section{a - Les éléments argyrophiles péribuccaux (fig. 4)}

$\mathrm{Si}$ l'on range les onze cercaires selon un ordre approximativement croissant de ces éléments (c'est-à-dire selon l'ordre évolutif cf. Richard 1971) on trouve : - deux Heterophyidae Haplorchiinae qui possèdent respectivement 12 et 18 éléments ; il s'agit d'Haplorchis taichui et H. pumilio, 
- deux Heterophyidae Apophallinae qui possèdent 19 et 20 éléments; il s'agit d'Apophallus muehlingi et Apophallus donicus,

- trois Acanthostomidae Acanthostominae, un Heterophyidae Euryhelminthinae et un Opisthorchiidae Opisthorchiinae chez lesquels on compte 18, 22 et 29 éléments pour Aphaloides coelomicola, Timoniella praeterita et $T$. imbutiforme (Acanthostomidae), 23 à 25 éléments pour Euryhelmis squamula (Euryhelminthinae) et 24 à 28 éléments pour Clonorchis sinensis (Opisthorchiinae),

- deux Heterophyidae Centrocestinae chez lesquels on compte 24 à 26 éléments pour Centrocestus formosanus, 28 à 34 éléments pour Centrocestus sp.

La division en familles n'est donc pas retrouvée en utilisant les caractéristiques du niveau $C_{I}$, de plus les diverses sous-familles d'Heterophyidae ne forment pas un ensemble homogène.

b - La chétotaxie caudale (fig. 5)

Si l'on range les espèces considérées selon le nombre décroissant de papilles caudales (c'est-à-dire selon l'ordre évolutif cf. Richard 1971) on trouve :

- deux Heterophyidae Apophallinae avec cinq paires de papilles caudales ; il s'agit d'Apophallus muehlingi et Apophallus donicus

- trois Acanthostomidae, trois Heterophyidae et un Opisthorchiidae avec quatre paires de papilles caudales; il s'agit de Timoniella imbutiforme, T. praeterita, Aphalloides coelomicola (Acanthostomidae Acanthostominae) Haplorchis taichui, Haplorchis pumilio (Heterophyidae, Haplorchiinae) Euryhelmis squamula (Heterophyidae, Euryhelminthinae) et Clonorchis sinensis (Opisthorchiidae, Opisthorchiinae),

- deux Heterophyidae Centrocestinae avec deux paires de papilles caudales; il s'agit de Centrocestus formosanus et Centrocestus sp.

La division en familles n'apparaît pas mieux avec la chétotaxie caudale qu'avec la chétotaxie céphalique ; l'hétérogénéité des Heterophyidae se manifeste à nouveau, la sous-famille la plus évoluée de cet ensemble paraissant être celle des Centrocestinae.

\section{4 - La chétotaxie permet la détermination de la superfamille des Opisthorchioidea}

Chez les Epitheliocystidia, le taxon suprafamilial est d'ordinaire facile à reconnaître par la chétotaxie des papilles céphaliques $\mathrm{C}_{\mathrm{I}} \mathrm{D}$ et, pour les cercaires leptocerques ou trichocerques, par la chétotaxie caudale, et, jusqu'à maintenant, il a toujours été possible, en considérant ces niveaux, d'attribuer une cercaire à une superfamille donnée.

Nous avons donc comparé la chétotaxie céphalique du cycle $\mathrm{C}_{\mathrm{I}}$ et la chétotaxie caudale des onze cercaires d'Opisthorchioidea à celles des cercaires de Plagiorchioidea et Allocreadioidea dont Bayssade-Dufour (1979) donne la liste pages 36, 40, 42 et 51. Chétotaxie céphalique du cycle $C_{\mathrm{I}}$

Les Plagiorchioidea et Allocreadioidea présentent au niveau du cycle $\mathrm{C}_{\mathrm{I}}$ une symétrie hexaradiée qui permet toujours de reconnaître :

- un petit nombre de papilles $\mathrm{C}_{\mathrm{I}} \mathrm{V}$ ( 1 chez les Plagiorchioidea, 1 à 3 chez les Allocreadioidea). 

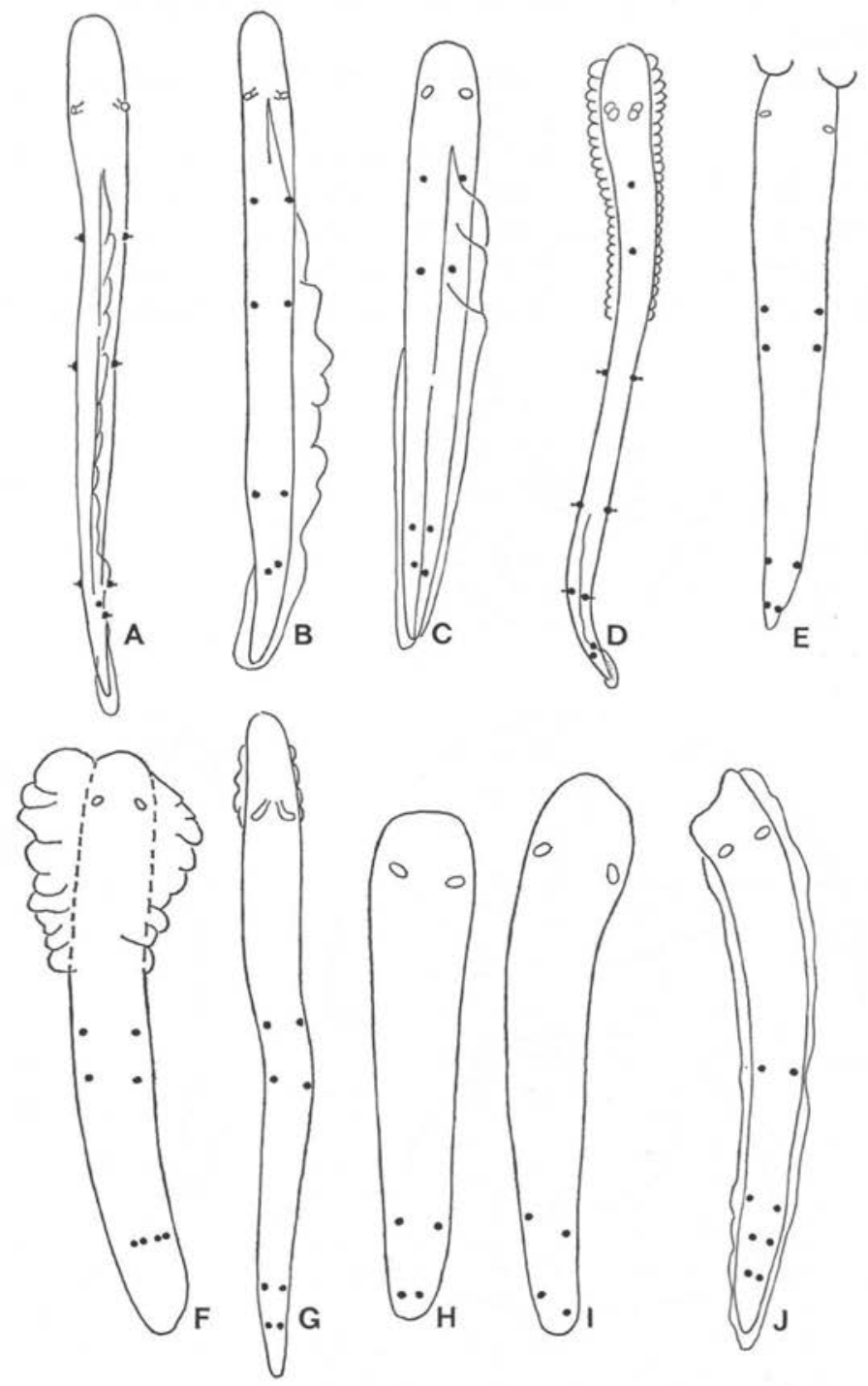

FIG. 5. - Chétotaxies caudales de cercaires d'Opisthorchioidea.

A : Timoniella imbutiforme d'après Maillard (1973); B : Timoniella praeterita ; C : Aphalloides coelomicola d'après Bayssade-Dufour et Maillard (1982); D : Apophallus muehlingi et Apophallus donicus d'après Odening (1973) ; E : Euryhelmis squamula d'après Combes, Jourdane et Richard (1974) ; F : Haplorchis pumilio d'après Bayssade-Dufour et Ow-Yang (I975) ; G : Haplorchis taichui; $\mathrm{H}$ et I : Centrocestus formosanus et Centrocestus sp. d'après Bayssade-Dufour, Albaret et Ow-Yang (1982); J : Clonorchis sinensis d'après Bayssade-Dufour, Albaret et Dong Cham Kim (1982). 
- un nombre variable de papilles $\mathrm{C}_{\mathrm{I}} \mathrm{L}$ (1 à $4 \mathrm{chez}$ les Plagiorchioidea, 1 à $8 \mathrm{chez}$ les Allocreadioidea),

- un petit nombre de papilles $\mathrm{C}_{\mathrm{I}} \mathrm{D}$ (2 chez les Plagiorchioidea, 1 chez les Allocreadioidea).

Il n'en est pas de même chez les Opisthorchioidea où seuls les éléments $\mathrm{C}_{\mathrm{I}} \mathrm{V}$ invaginés sont identifiables avec certitude; les autres éléments argyrophiles péribuccaux forment des séries continues ou discontinues qui ne se localisent plus aux seules intersections des six axes, $\mathrm{V}, \mathrm{L}$ et $\mathrm{D}$ avec la première commissure céphalique. La symétrie hexaradiée originelle ne se manifeste plus au niveau $\mathrm{C}_{\mathrm{I}}$ chez les Opisthor-
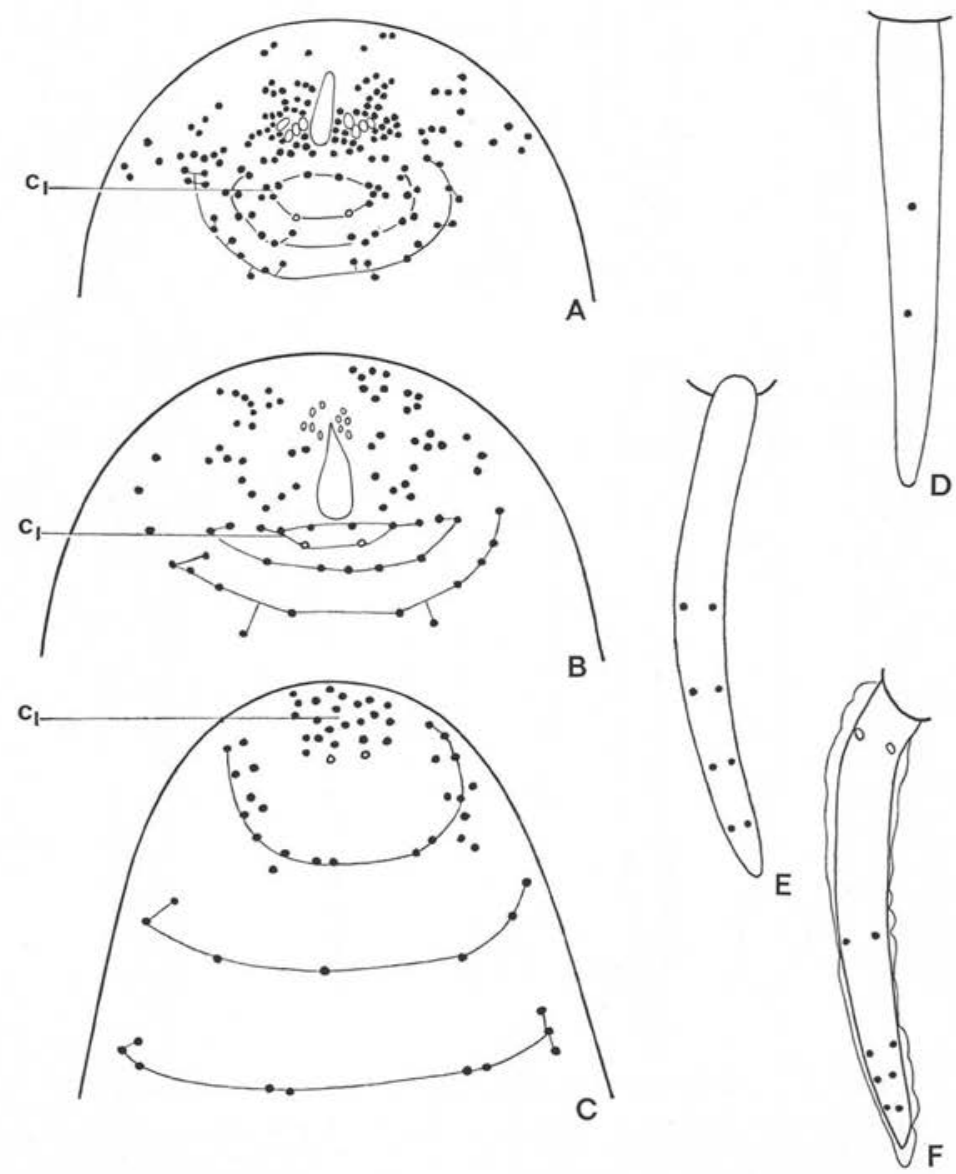

FIG. 6. - A, B, C : Chétotaxies céphaliques d'Epitheliocystidia;

D, E, F : Chétotaxies caudales d’Epitheliocystidia.

A et D : Chétotaxies de Paralepoderma progeneticum, Plagiorchioidea; B et E : Chétotaxies de Macrolecithus papilliger, Allocreadioidea ; C et F : Chétotaxies de Clonorchis sinensis, Opisthorchioidea. 
chioidea et ce caractère permet de les distinguer de l'ensemble formé par les Plagiorchioidea et Allocreadioidea (fig. $6, A, B, C ; A$ d'après Monod 1977; $B$ d'après Lambert 1972; $C$ d'après Bayssade-Dufour et coll. 1982).

\section{Chétotaxie caudale}

Les Plagiorchioidea portent une paire de papilles caudales. Les Allocreadioidea leptocerques ou trichocerques portent un nombre variable, de papilles caudales et, à l'exception des Rhodométopes et des Allocreadiidae, ce nombre est toujours différent de celui des Opisthorchioidea (cf. Bayssade-Dufour 1979 fig. 29 et 30). Les Opisthorchioidea portent, ainsi que nous l'avons vu ci-dessus, deux à cinq paires de papilles U. Leur chétotaxie caudale ne permet donc pas de les reconnaître d'une Rhodométope ou d'un Allocreadiidae qui porte quatre paires de papilles $\mathrm{U}$; il faut, pour ces deux familles faire appel à un autre élément de la morphologie : les pores excréteurs caudaux; ils sont toujours absents chez les Rhodométopes et Allocreadiidae, toujours présents chez les Opisthorchioidea. Les Opisthorchioidea se caractérisent donc par deux à cinq paires de papilles $U$ sur une queue pourvue de pores excréteurs (fig. 6, D, E, F; D : d'après Monod 1977, $F$ : d'après Lambert 1972, $E$ : d'après Bayssade-Dufour et coll. 1982).

Les résultats exposés ci-dessus concernent onze cercaires d'Opisthorchioidea; ils sont donc insuffisants pour des conclusions définitives sur cette superfamille. Toutefois, l'absence de correspondance entre la chétotaxie et les divisions en familles actuellement admises, l'absence d'homogénéité dans la chétotaxie des divers Heterophyidae, les controverses sur le contenu des Opisthorchioidea et des Heterophyidae (cf. Skrjabin 1952, Yamaguti 1971) manifestent la complexité de ces questions et semblent indiquer que la systématique des Opisthorchioidea, fondée sur certains caractères morphologiques d'adultes, n'est pas naturelle.

\section{BIBLIOGRAPHIE}

BAyssade-Dufour Ch. : L'appareil sensoriel des cercaires et la systématique des Trématodes Digénétiques. Mém. Mus. Nat. Hist. Nat., A Zool., 1979, I13, I-81.

Bayssade-Dufour Ch., Albaret JL., Dong Cham KIm : Chétotaxie cercarienne d'une souche coréenne de Clonorchis sinensis. Présence vraisemblable de ce Trématode en Afrique. Ann. Parasitol. Hum. Comp., 1982, 57, 361-366.

Bayssade-Dufour Ch., Albaret J. L. et Ow-Yang C. K. : Sensilles et protonéphridies des cercaires de Centrocestus formosanus et Centrocestus sp. (Centrocestinae, Heterophyidae). Ann. Parasitol. Hum. Comp., 1982, s7, 565-572.

BAysSAde-Dufour Ch. et Marllard C. : Discussion sur la position taxonomique d'Aphalloides coelomicola Dollfus, Chabaud et Golvan 1957 (Trematoda, Opisthorchioidea). Ann. Parasitol. Hum. Comp., I982, $57,549-553$.

BAySSADE-DUFOUR Ch., OW-YANG C. K. : The sensory receptors of two cercariae from Malaysia : Trichobilharzia brevis (Schistosomatidae) and Haplorchis pumilio (Heterophyidae). The Southeast Asian J. Trop. Med. Pub. health, 1975, 6, 338-342.

Combes C., BAyssade-Dufour Ch., CAssone J. : Sur l'imprégnation et le montage des cercaires pour l'étude chétotaxique. Ann. Parasitol. Hum. Comp., 1976, sI, 399-400.

COMBEs C., JouRdANe J., RICHARD J. : Recherches sur le cycle biologique d'Euryhelmis squamula (Rudolphi, 1819) parasite de Neomys fodiens dans les Pyrénées. Z. Parasitkde, 1974, 44, 81-92.

FAust E. C., Nishigori M. : The life cycles of two new species of Heterophyidae, parasitic in Mammals and Birds. J. Parasitol., I926, I3, 91-127.

LAMBERT A. : Étude de quelques plathelminthes parasites de Cyprinidae en Languedoc-Roussillon. Thèse Doct. Spécial. Acad. Montpellier, 1972, 133 p. polycopiées. 


\section{Ch. BAYSSADE-DUFOUR, J.-L. ALBARET ET C. K. OW-YANG}

LA Rue G. R. : The Classification of Digenetic Trematoda : a review and a new system. Expl. Parasitol., 1957, 6, 306-344.

MAILlARD C. : Étude du cycle évolutif du Trématode Acanthostomum imbutiforme (Molin, I859) Gohar, 1934, parasite de Morone labrax (Linné, 1758). Ann. Parasitol. Hum. Comp., 1973,

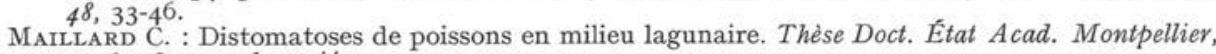
I976, 383 p. polycopiées.

Monop R.: Étude de l'helminthofaune des couleuvres du genre Natrix dans le sud de la France. Biologie et écologie de la progenèse chez les Digènes du genre Paralepoderma. Thèse Doct. Spécial. Acad. Montpellier, 1977, 262 p. polycopiées.

Odening K. : Der Lebenszyklus des Trematoden Apophallus donicus in Berlin im Vergleich zu A. muehlingi. Biol. Z $\mathrm{bl}$., 1973, 92, 455-494.

Richard J. : La chétotaxie des cercaires. Valeur systématique et phylétique. Mem. Mus. Nat. Hist. Nat., A. Zool., 1971, 67, I-I79. 